\title{
Use of Flow Cytometry to Rapidly Optimize the Transfection of Animal Cells
}

BioTechniques 28:148-154 (January 2000)

\author{
Michelle B.A. Keith, Patrick J. \\ Farrell, Kostas Iatrou and Leo \\ A. Behie \\ The University of Calgary, \\ Calgary, AB, Canada
}

\section{INTRODUCTION}

Transfection of an expression cassette into cultured animal cells is an important tool for investigating transient heterologous protein expression and is also the first step in the generation of stably transformed cells lines. We have previously reported that high yields of recombinant proteins can be obtained from stably transformed lepidopteran insect cells $(6,7)$ and anticipate that improvements could be achieved by improved transfection protocols. Various methods of gene transfer have been developed, including electroporation (17), DEAE dextran transfection (16), calcium phosphate precipitation $\left(\mathrm{CaPO}_{4}\right)(11,13,21)$ and cationic liposomes such as lipofectin $(4,8-10,14$, $15,19)$. Although in some cases toxicity limits its use, lipid-mediated transfection was shown to be 5-100-fold more efficient than methods using $\mathrm{CaPO}_{4}$ or DEAE dextran (9). The wide use of cationic liposomes is also due to the ease of the transfection procedure and the high frequency of stable gene expression (18). With the addition of cationic liposomes to a DNA solution, a lipid/DNA complex spontaneously forms. When added to cell cultures, the DNA is transported into the cells, either by endocytosis (22) or by fusion with the plasma membrane (2), and the exogenous DNA migrates to the nucleus.

Two important parameters to be examined and optimized within the transfection procedure are the heterologous protein expression level and the transfection efficiency. Measuring these two parameters is often done using a transfection marker or reporter gene. Quantification of the expression level using reporter proteins such as $\beta$-galactosidase ( $\beta$-gal) $(1,11,14,15,18,21)$, luciferase
$(5,11)$ and CAT $(12,20)$ requires a series of time-consuming steps that may involve cell lysis, soluble protein extraction and quantification, completion of an assay with expensive substrates and spectrophotometer analysis (18). There are two further disadvantages to this technique: $(i)$ assaying the protein activity of transfected cells' extracts is only an indirect measurement of gene expression and (ii) the simultaneous measurement of the transfection efficiency and expression level is not possible.

Determination of the transfection efficiency using the above mentioned reporter proteins can also be tedious. For example, the use of $\beta$-gal involves timeconsuming steps, including cell fixation, staining, incubation, photography and calculating the number of stained cells (21). In contrast, green fluorescence protein (GFP) fluorescence can be noninvasively detected in living cells. Flow cytometric analysis of GFP expression has become a popular means of gene transfer quantification $(4,19)$ because this technique permits the simultaneous measurement of the protein expression level and transfection efficiency without the requirement for cell fixation or sample preparation. Approximately $60 \mathrm{~h}$ posttransfection, cells simply suspended in PBS can be analyzed by flow cytometry to rapidly determine both the percentage of positive cells above autofluorescence and the expression level of GFP in each individual cell.

This report examines the effect of varying several parameters within the transfection protocol used by Farrell et al. $(6,7)$ for ultimately improving the expression level of recombinant proteins from transformed lepidopteran insect cells. To devise an improved transfection protocol using the cationic liposome lipofectin, those parameters reported to 
influence the transfection efficiency and cellular expression level were systematically investigated: the lipid/DNA incubation temperature, lipid incubation time, lipid/DNA incubation time, lipid and DNA concentration, incubation vessel and transfection duration. The experimental aim of this report is to develop a protocol that achieves the highest possible number of transfected insect cells with the greatest expression level of heterologous protein. Most of these variables are not species specific and may be applied to other insect cell lines and even other animal cell lines.

\section{MATERIALS AND METHODS}

\section{Plasmid Constructions}

The lepidopteran expression cassette overexpressing the Aequorea victoria GFP, pIE1/153A.gfp, has been described previously (7).

\section{Cell Culture and Transfections}

Bm5 and Sf-21 cells were cultured in IPL-41 insect medium (Life Technologies, Gaithersburg, MD, USA) supplemented with $0.2 \mathrm{~g} / \mathrm{L}$ L-glutamine, $2.6 \mathrm{~g} / \mathrm{L}$ tryptose phosphate, 9.0 $\mathrm{g} / \mathrm{L}$ sucrose, $0.35 \mathrm{~g} / \mathrm{L}$ sodium bicarbonate, $0.069 \mathrm{mg} / \mathrm{L}$ zinc sulphate, 7.59 $\mathrm{mg} / \mathrm{L}$ aluminum potassium sulphate, $0.5 \mathrm{~g} / \mathrm{L}$ calcium chloride and $10 \%$ fetal bovine serum (FBS; JRH Biosciences, Lenexa, KS, USA). For all transfection experimental investigations, cells were seeded into Falcon ${ }^{\circledR}$ 6-well plates (Becton Dickinson Vacutainer Systems, Franklin Lakes, NJ, USA) at a density of $5 \times 10^{5}$ cells $/ \mathrm{mL}(2 \mathrm{~mL} /$ well $)$ and allowed to adhere overnight (most cultured lepidopteran cells are weakly adherent). Cells were then transfected with the plasmid pIE1/153A.gfp. The generic transfection protocol for a single well is as follows. We prepared the transfection solution by first incubating
$30 \mu \mathrm{g} / \mathrm{mL}$ of LIPOFECTIN ${ }^{\circledR}$ Reagent (Life Technologies) in $275 \mu \mathrm{L}$ of basal medium on ice for $30 \mathrm{~min}$ in Falcon polystyrene conical tube vessels (Becton Dickinson Vacutainer Systems). We added this solution to $6 \mu \mathrm{g} / \mathrm{mL}$ of plasmid DNA in $275 \mu \mathrm{L}$ of basal medium, and the mixture was further incubated on ice for $15 \mathrm{~min}$. After removing the serum-containing medium and rinsing the wells with basal medium, we added the transfection solution to the cells. Following transfection for $5 \mathrm{~h}$ at $28^{\circ} \mathrm{C}$, we removed the transfection solution, rinsed the cells to remove residual transfection solution and added $2 \mathrm{~mL}$ of complete medium to the cells. After $60 \mathrm{~h}$, we verified the cells to be over $90 \%$ viable by trypan blue staining before flow cytometric analysis.

\section{Flow Cytometry}

Suspended cell samples were diluted fivefold in polystyrene tubes that con-
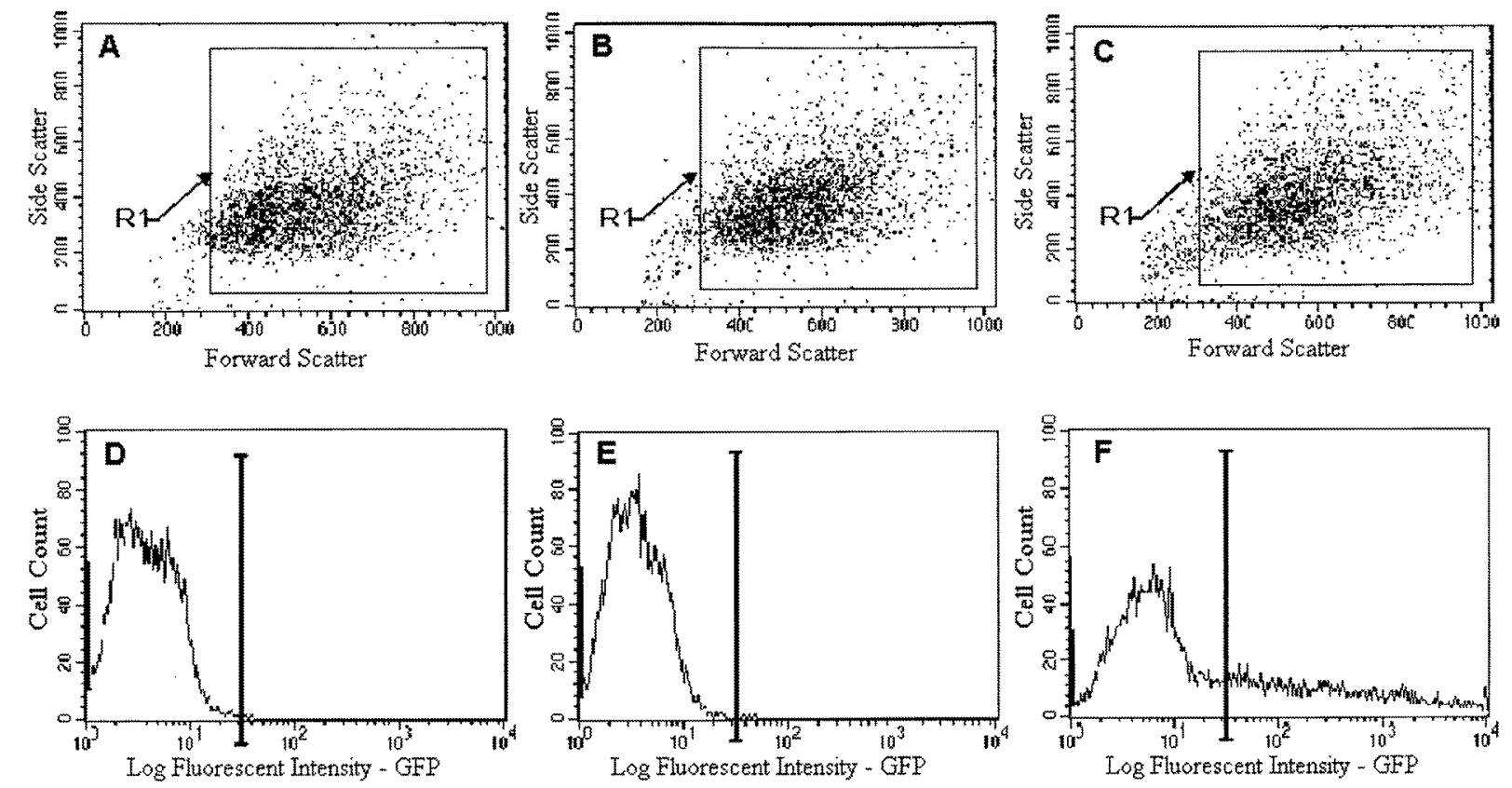

Figure 1. Evaluation of control preparation methods and determination of transfection efficiency and mean GFP expression of Sf-21-transfected cells. Two control samples were prepared: one nontransfected control and one control transfected with a blank vector (expression vector minus GFP). The scatter pattern of cells was isolated from debris by setting the gate R1 for the nontransfected control (A), the blank vector control (B) and the GFP-transfected (C) cells. Based on the cells gated in the region R1, the histograms of nontransfected control (D) and blank vector control (E) illustrate the autofluorescence of the Sf-21 cells. As indicated by the histograms of these two controls, the lipofectin did not induce additional autofluorescence. The autofluorescence of the cells was gated, as shown by the vertical line (D, E and F). Within the histogram of GFP-transfected cells (F), the region to the right of the vertical line represents the GFP fluorescence. The transfection efficiency and mean expression level can then be calculated by the Lysys software. 
tained PBS and directly introduced into the flow cytometer. A FACScan ${ }^{\mathrm{TM}}$ (Becton Dickinson Immunocytometry Systems, San Jose, CA, USA) equipped with a $15 \mathrm{~mW}, 488 \mathrm{~nm}$, argon-ion laser recorded forward scatter (FSC), side scatter (SSC) and green fluorescence (FL1). FSC and SSC data were used to identify viable cells, and gates were set to exclude cellular debris. We collected data from 10000 events. Transfection and mean expression were determined by subtracting the autofluorescent FL1 signal of the control cells from the FL1 of the transfected cell population using Lysys $^{\mathrm{TM}}$ software (Becton Dickinson Immunocytometry Systems). The FL1 signal was recorded on a logarithmic scale and converted to linear units using Equation 1, as described by the software manufacturer. For our system, the scaling factor used was 64.

Linear mean channel number $=$

10 (log mean channel number)/scaling factor [Eq. 1]

\section{RESULTS}

\section{Analysis of Transfection Results Using Flow Cytometry}

To account for a cell line's natural fluorescent signal, a control of wildtype cells was prepared simultaneously with the cells to be transfected. For each experiment in this study, one well of the 6-well plate was assigned as a control, and its cells were therefore not transfected. The inoculum cells for both the control and transfection were derived from the same culture to eliminate differences between the growth cycle and other culture conditions. The autofluorescence of wild-type cells was also measured by the flow cytometer 60 $\mathrm{h}$ posttransfection, immediately following the fluorescent intensity measurement of the GFP-transfected cells.

Because lipid-based transfection reagents can influence the natural fluorescence of cells, the conventional method of control preparation involves lipidmediated transfection of the cells with a blank vector. Figure 1 compares the two control preparation methods and shows that lipofectin had no effect on the autofluorescence of the Sf-21 cells. An identical study using Bm5 cells for the two control samples yielded a matching result (data not shown). Figure 1 also indi-
A

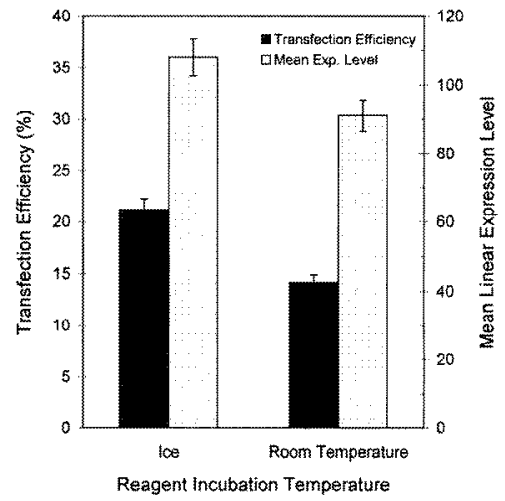

B

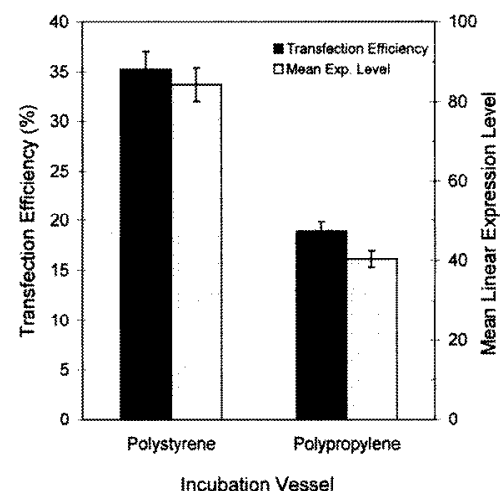

Incubation Vessel
C

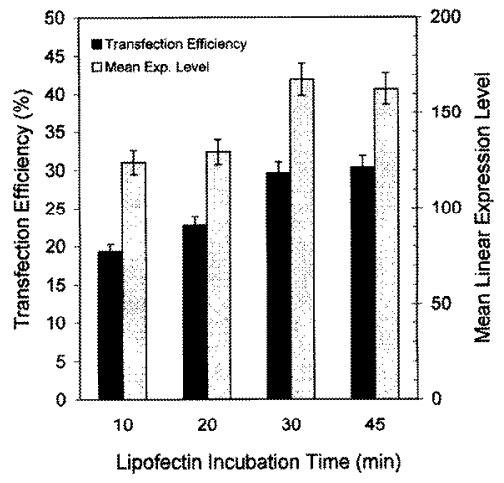

E

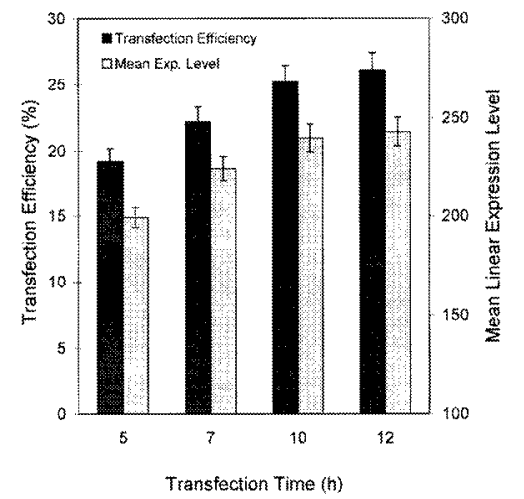

Figure 2. Optimization of 5 parameters in the transfection protocol of lepidopteran insect cells. Graphs A-E represent the results of transfections involving the varying of the incubation temperature, the incubation vessel, the lipid incubation time, the lipid/DNA incubation time and the transfection time, respectively, while holding the remainder of the parameters in the generic transfection protocol constant. One million cells were transfected in each experiment with a reporter plasmid expressing GFP. Sixty hours after transfection, cells were suspended in PBS and analyzed using flow cytometry to determine the transfection efficiency and mean cellular expression level. Duplicate samples were analyzed. 
cates the manner in which the control cells' autofluorescent signal was subtracted from the fluorescent signal of the Sf-21 transfected cells. Single cells were isolated from debris based on their SSC vs. FSC characteristics (Figure 1, A, B and $\mathrm{C}$ ). The frequency histograms of the control and transfected cells show the profiles of autofluorescence and green fluorescence, respectively (Figure 1, D,

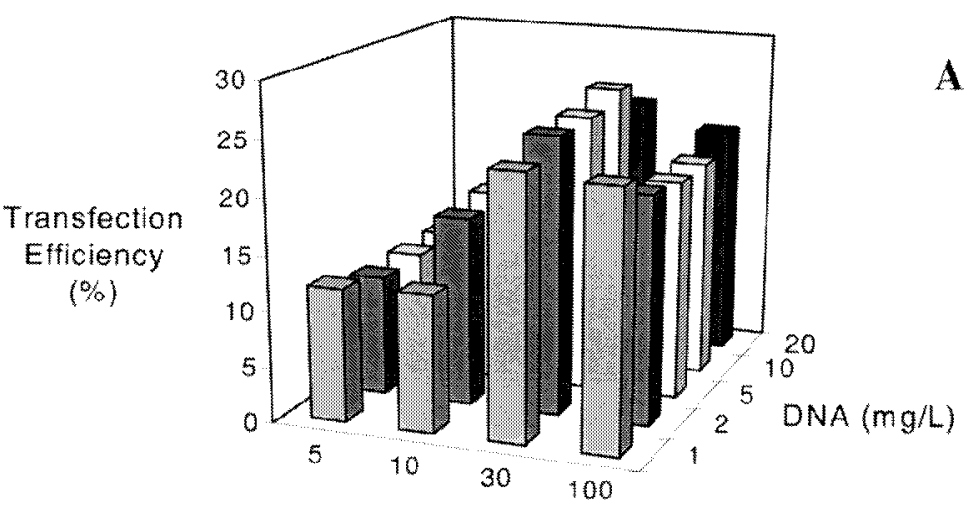

A

Lipofectin $^{R}$ Reagent

$(\mathrm{mg} / \mathrm{L})$

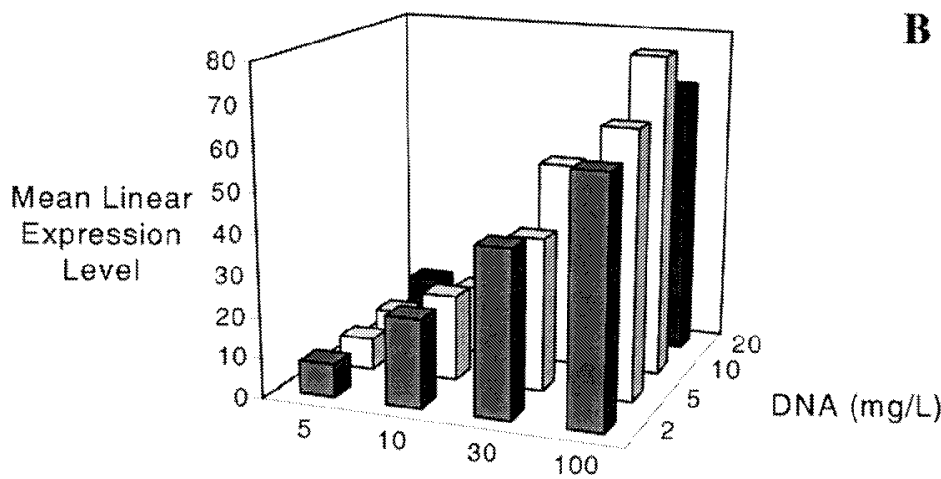

$\mathbf{B}$

Lipofectin ${ }^{\text {A Reagent }}$

$(\mathrm{mg} / \mathrm{L})$

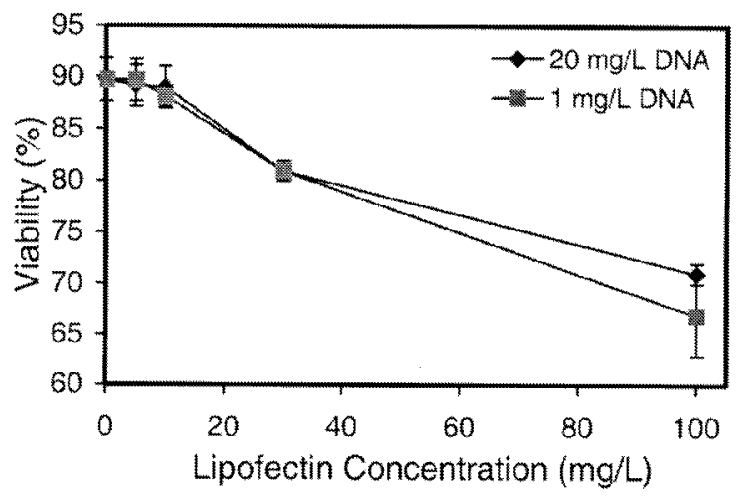

C

Figure 3. Effect of varying the lipofectin/DNA concentrations in the transfection solution on the transfection efficiency $(A)$, the mean expression level $(B)$ and the cell viability $(C)$ using $B m 5$ cells. The transfection efficiencies and mean cellular expression levels were determined using flow cytometric analysis. Error inherent to flow cytometry analysis is less than 5\%. 
E and F). Subtraction of the autofluorescence signal from the transfected cell population allowed the calculation of the transfection efficiency and mean GFP expression level. Duplicate samples were analyzed.

\section{Optimization of Generic Protocol}

Figure 1 shows the base case transfection results for Sf-21 cells, in which the transfection protocol described by Farrell et al. (7) was used. Using the analysis technique indicated by Figure
1 and the generic transfection protocol, several parameters that impact the transfection efficiency and expression level of transfected cells were investigated to devise an improved transfection protocol. Hence, the purpose of this study was to illustrate the ease with which the flow cytometric analysis technique facilitates the optimization of an animal cell transfection protocol. Results of this study provide a useful protocol for the transfection of lepidopteran insect cells.

The lipofectin manufacturer's proto-

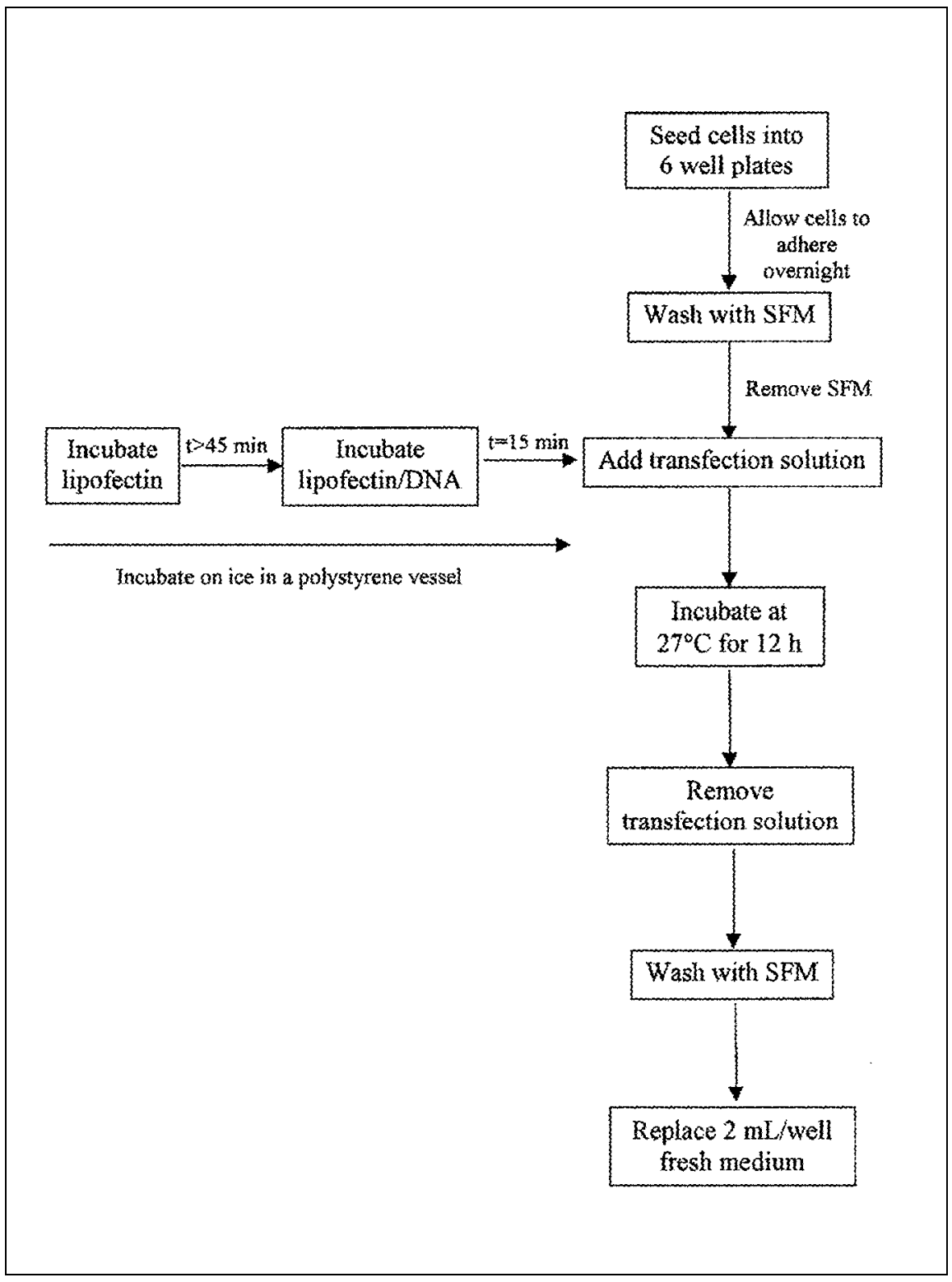

Figure 4. Improved protocol for the transfection of lepidopteran insect cells. SFM refers to serumfree or basal medium. For the experimental investigations described in this report, flow cytometry analysis was carried out $60 \mathrm{~h}$ after the removal of the transfection solution. col suggests that the lipofectin/DNA incubation should be carried out at room temperature; however, we investigated whether the lipid/DNA complex formation might be improved by incubation on ice. The temperature at which the incubation of reagents should take place was investigated and, using Bm5 cells, it was determined that incubation of the lipofectin and lipofectin/DNA solution on ice $\left(0^{\circ} \mathrm{C}\right)$ yielded an improved mean GFP expression level and a transfection efficiency of $21 \%$ compared with the $14 \%$ achieved with incubation at room temperature (Figure 2A). The temperature shock did not appear to harm the transfected cells (trypan blue staining).

Because we had previously observed that the use of a specific incubation vessel affected the transfection results, we compared the use of conical tubes composed of polystyrene and polypropylene (Becton Dickinson Vacutainer Systems). Using Sf-21 cells, Figure $2 \mathrm{~B}$ shows that the polymer material from which the tube was manufactured greatly influenced the results. Use of polystyrene tubes yielded both a much higher mean GFP expression level and a transfection efficiency of $35 \%$ compared with the $18 \%$ obtained with the polypropylene tubes. This result indicates that the formation of lipid/DNA complexes may have been inhibited when incubation took place in the polypropylene tubes.

Preincubation of the lipid in basal medium is recommended by the manufacturer for at least $30 \mathrm{~min}$ before the addition of diluted DNA. The effect of lipofectin incubation has previously been reported for BHK-21 cells (4). To observe the effect on Bm5 insect cells, the diluted lipofectin solution was incubated for 10, 20, 30 and $45 \mathrm{~min}$. It was observed that a longer lipid incubation of at least $30 \mathrm{~min}$ corresponds to both a higher mean expression level and transfection efficiency greater than $30 \%$ (Figure 2C). These results conform to those previously reported (4), suggesting that a longer lipofectin incubation dramatically improves the formation of lipid/DNA complexes.

Incubation of the diluted lipofectin with the diluted DNA permits the formation of lipid/DNA complexes. To determine the optimal incubation time, five lipofectin/DNA incubation times were 
examined, ranging from 7.5-17.5 min. Figure 2D shows that incubation for less than 15 min yielded lower transfection efficiencies, indicating that insufficient time had likely elapsed for the formation of lipid/DNA complexes. An incubation time of 15 min yielded the highest transfection efficiency of $27 \%$. Incubation for longer than 15 min caused a slight decrease in the transfection efficiency.

The length of time cells were exposed to the transfection solution (the transfection duration) was examined over periods of 5, 7, 10 and $12 \mathrm{~h}$. Both the transfection efficiency and mean expression level increased as the transfection duration increased (Figure 2E). Transfection durations of more than 10 $\mathrm{h}$ were advantageous. However, some of the cells became detached from the adherent surface and were lost during the subsequent removal of transfection solution and rinsing of the cells. Fur- thermore, prolonged exposure to lipids has a toxic effect on some cells (13).

By varying the lipofectin and plasmid DNA concentrations from 5-100 $\mu \mathrm{g} / \mathrm{mL}$ and $1-20 \mu \mathrm{g} / \mathrm{mL}$, respectively, we investigated the effect of lipofectin/ DNA concentration in the transfection solution. Figure 3 reveals that the lower lipid concentrations of 5 and $10 \mu \mathrm{g} / \mathrm{mL}$ yielded transfection efficiencies below $16 \%$ and low mean expression levels. Concentrations of 30 and $10 \mu \mathrm{g} / \mathrm{mL}$ of lipofectin and DNA, respectively, yielded the highest transfection efficiency of $26 \%$. We observed a decrease in transfection efficiency at the highest lipid concentration of $100 \mu \mathrm{g} / \mathrm{mL}$. These levels of lipid are toxic to cells (13) in addition to being uneconomical.

The toxic effect of lipids in high concentration after prolonged exposure was verified by transfecting Bm5 cells with solutions containing lipofectin concentrations of $5-100 \mu \mathrm{g} / \mathrm{mL}$ and DNA concentrations of 1 and $20 \mu \mathrm{g} /$ $\mathrm{mL}$. Following the generic transfection protocol, the cells were exposed to the transfection solution for $5 \mathrm{~h}$, and cell viabilities were determined approximately $60 \mathrm{~h}$ posttransfection using trypan blue exclusion. Figure 3 indicates that the viability decreased sharply as the lipid concentration increased above $10 \mu \mathrm{g} / \mathrm{mL}$. Over the range of lipid concentrations, similar results were obtained with the 20 and $1 \mu \mathrm{g} / \mathrm{mL}$ DNA concentrations, suggesting that a substantial increase in DNA concentration is not toxic to the cells.

\section{DISCUSSION}

Here, we present an analysis of those parameters influencing the efficacy of animal cell transfection. The 
analysis used GFP as a reporter protein, which allowed for the rapid and simultaneous determination of the transfection efficiency and mean expression by flow cytometry. This analysis has led to an improved transfection protocol for lepidopteran insect cells, which can now be used to test transient gene expression in different cell lines, compare different expression vectors or generate stably transformed insect cell lines.

Based on the results given in this report, Figure 4 illustrates this improved transfection protocol. Variables shown to impact the efficiency of gene transfer included the incubation temperature, the incubation vessel, the incubation time, the transfection time and the amounts of DNA and lipid. The improved transfection protocol described here has now been successfully used to transfect nine insect cell lines derived from such species as Bombyx mori, Trichoplusia ni, Mamestra brassicae and Lymantria dispar with GFP (data not shown). Implementing the improved protocol caused an increase in transfection efficiency from $25 \%$ to $40 \%$ and $24 \%$ to $40 \%$ with Bm5 and Sf-21 cells, respectively, and equally dramatic increases in the mean expression levels (data not shown).

For lipid-mediated transfection, the aim of transfection optimization is to increase the lipid/DNA complex-cell contact, which leads to an increased transfection efficiency. To further optimize the protocol for a specific cell line, the optimal cell density and time during the growth cycle for transfection should be determined because actively dividing cells are generally transfected more efficiently than resting cells (15). Along with optimizing the parameters discussed here, improved results have also been reported by limiting the transfection solution volume and directly centrifuging the lipid/DNA complex onto the target cells (20).

\section{ACKNOWLEDGMENTS}

The authors wish to acknowledge the generous financial support from the Natural Sciences and Engineering Research Council of Canada, the Medical Research Council of Canada and the Alberta Heritage Foundation for Medical Research.

\section{REFERENCES}

1.Alam, J. and J.L. Cook. 1990. Reporter genes: application to the study of mammalian gene transcription. Anal. Biochem. 188:245254.

2.Bangham, A.D. 1992. Liposomes: realizing their promise. Hosp. Pract. 27:51-62.

3.Cheng, L., J. Fu, A. Tsukamoto and R.G. Hawley. 1996. Use of green fluorescent protein variants to monitor gene transfer and expression in mammalian cells. BioTechnology 14:606-609.

4.Ciccarone, V. and P. Hawley-Nelson. 1995. Lipofectin ${ }^{\circledR}$ transfection activity increased by protocol improvement. Focus 17:103.

5.de Wet, J.R., K.V. Wood, M. DeLuca, D.R. Helinski and S. Subramani. 1987. Firefly luciferase gene: structure and expression in mammalian cells. Mol. Cell. Biol. 7:725-737.

6.Farrell, P.J., L.A. Behie and K. Iatrou. 1999. Transformed lepidopteran insect cells: new sources of recombinant human tissue plasminogen activator. Biotechnol. Bioeng. 64:426-433.

7.Farrell, P.J., M. Lu, J. Prevost, C. Brown, L.A. Behie and K. Iatrou. 1998. High-level expression of secreted glycoproteins in transformed lepidopteran insect cells using a novel expression vector. Biotechnol. Bioeng. 60:656-663.

8.Felgner, J.H., R. Kumar, C.N. Sridhar, C.J. Wheeler, T.J. Tsai, R. Border, P. Ramsey, M. Martin and P.L. Felgner. 1994. Enhanced gene delivery and mechanism studies with a novel series of cationic lipid formulations. J. Biol. Chem. 4:2550-2561.

9.Felgner, P.L, T.R. Gadek, M. Holm, R. Roman, H.W. Chan, M. Wenz, J.P. Northrop, G.M. Ringold and M. Danielsen. 1987. Lipofection: a highly efficient, lipid-mediated DNA-transfection procedure. Proc. Natl. Acad. Sci. USA 84:7413.

10.Felgner, P.L. and G.M. Ringold. 1989. Cationic liposome-mediated transfection. Nature 337:387-388.

11.Gaunitz, F., M. Papke and R. Gebhardt. 1996. Transient transfection of primary cultured hepatocytes using CaPO4/DNA precipitation. BioTechniques 20:826-830.

12.Gorman, C.M., L.F. Moffat and B.H. Howard. 1982. Recombinant genomes which express chloramphenicol acetyltransferase in mammalian cells. Mol. Cell. Biol. 2:10441051.

13.Graham, F.L. and A.J. van der Eb. 1973. A new technique for the assay of infectivity of human adenovirus 5 DNA. Virology 52:456.

14.Griffiths, T., M. Russell, K. Froning, B.D. Brown, S.M. Scanlon, M. Almazan, R. Marcil and J.P. Hoeffler. 1997. The PerFect ${ }^{\mathrm{TM}}$ Lipid Optimizer Kit for maximizing lipid-mediated transfection of eukaryotic cells. BioTechniques 22:982-987.

15.Lascombe, I., P. Mougin, C. Vuillermoz, G.L. Adessi and M. Jouvenot. 1996. Gene transfer into subcultured endometrial cells using lipofectin. BioTechniques 20:88-91.

16.McCutchan, J.H. and J.S. Pagano. 1968. Enhancement of the infectivity of simian virus 40 deoxyribonucleic acid with diethylaminoethyldextran. J. Natl. Cancer Inst. 42:351-357.
17.Neumann, E., M. Schaefer-Ridder, Y. Wang and H. Hofschneider. 1982. Gene transfer into mouse lyoma cells by electroporation in high electric fields. EMBO J. 1:841-845.

18.Teifel, M. and P. Friedl. 1995. New lipid mixture for efficient lipid-mediated transfection of BHK cells. BioTechniques 19:79-82.

19.Tseng, W., F.R. Haselton and T.D. Giorgio. 1997. Transfection by cationic liposomes using simultaneous single cell measurements of plasmid delivery and transgene expression. $\mathrm{J}$. Biol. Chem. 272:25641-25647.

20.Verma, R.S., D. Giannola, W. Shlomchik and S.G. Emerson. 1998. Increased efficiency of liposome-mediated transfection by volume reduction and centrifugation. BioTechniques 25:46-49.

21.Zhang, G. and S.R. Kain. 1996. Transfection maximizer increases the efficiency of calcium phosphate transfections with mammalian cells. BioTechniques 21:940-945.

22.Zhu, N. and L. Huang. 1994. DNA transfection mediated by cationic liposomes containing lipopolylysine: characterization and mechanism of action. Biochim. Biophys. Acta 1189:195-203.

Received 10 May, 1999; accepted 5 August 1999.

Address correspondence to:

Leo A. Behie

The Department of Chemical Engineering

The University of Calgary

Calgary, AB, T2N 1N4, Canada

Internet:behie@ucalgary.ca 